S. Kebekus

Nagoya Math. J.

Vol. 157 (2000), 149-176

\title{
RELATIVELY MINIMAL QUASIHOMOGENEOUS PROJECTIVE 3-FOLDS
}

\author{
STEFAN KEBEKUS
}

\begin{abstract}
In the present work we classify the relatively minimal 3-dimensional quasihomogeneous complex projective varieties under the assumption that the automorphism group is not solvable. By relatively minimal we understand varieties $X$ having at most $\mathbb{Q}$-factorial terminal singularities and allowing an extremal contraction $X \rightarrow Y$ where $\operatorname{dim} Y<3$.
\end{abstract}

\section{$\S 1$. Introduction}

Let $X$ be a smooth projective threefold and $G$ a connected algebraic group acting algebraically on $X$. By minimal model theory, there exists a sequence of extremal ray contractions and flips such that the resulting variety $X^{\prime}$ has at most $\mathbb{Q}$-factorial terminal singularities and either the canonical sheaf $K_{X^{\prime}}$ is numerically effective, or $X^{\prime}$ allows an extremal ray contraction of fiber type. See the introductory chapter of [KMM87] for a detailed account of these matters.

It has been shown in [Keb98] that all steps of the minimal model program are equivariant with respect to the action of $G$. If one assumes additionally that $G$ acts almost transitively, which is to say that the $G$-action has an open orbit, then it is shown that the minimal model program always ends with a contraction of fiber type. The aim of the present paper is a classification of these varieties, more precisely, a classification of 3-folds which are quasihomogeneous under the action of a linear, non-solvable algebraic group and relatively minimal in the sense of the following definition

Definition 1.1. Throughout the present paper, a relatively minimal variety $X^{\prime}$ over a base $Y$ is a projective variety with at most $\mathbb{Q}$-factorial terminal singularities which has an extremal ray contraction $\phi: X^{\prime} \rightarrow Y$ of fiber type, i.e., $\operatorname{dim} Y<\operatorname{dim} X$.

Received May 19, 1998.

The author was supported by scholarships of the Graduiertenkollegs "Geometrie und mathematische Physik" and "Komplexe Mannigfaltigkeiten" of the Deutsche Forschungsgemeinschaft 
It should be remarked that this notion is not commonly accepted and that other authors use different definitions.

The answer to the analogous problem in dimension 2 is known since decades: since a surface which is quasihomogeneous under the action of a linear algebraic group is always rational, the only varieties are $\mathbb{P}_{2}$ and Hirzebruch-surfaces $\Sigma_{n}$. The succeeding Main Theorem should be seen as a direct generalization of this to dimension 3. See [MU83] for a different approach to this kind of questions.

We now list concrete examples which occur in the classification. Notation: a "Zariski $\mathbb{P}_{r}$-bundle" is a variety of the form $\mathbb{P}(E)$, where $E$ is a vector bundle. Call $\mathbb{P}(E)$ "splitting" if $E$ is a direct sum of line bundles: $E=\bigoplus_{i=1}^{r} L_{i}$

- Homogeneous threefolds such as $\mathbb{P}_{3}$, the quadric $\mathbb{Q}_{3}$ and the full flag variety $F_{(1,2)}(3)$.

- The $S L_{2}$-quasihomogeneous Fano-manifolds described by Mukai and Umemura in [MU83]. In Iskovskih's list of Fano-threefolds (see e.g. [Isk83, Thm. 1]) they appear under the name $A_{22}^{\prime}$ and $B_{5}$. Other customary names are $V_{22}^{S}$ and $V_{5}$, respectively.

- The weighted projective spaces $\mathbb{P}_{(1,1,2,3)}$ and $\mathbb{P}_{(1,1,1,2)}$. The first space is described in detail in [Keb99, Ex. 4.1], the latter is the blow-down of the negative section of $\mathbb{P}\left(\mathcal{O}_{\mathbb{P}_{2}}(2) \oplus \mathcal{O}_{\mathbb{P}_{2}}\right)$.

- Varieties over $Y \cong \mathbb{P}_{1}$ which are locally isomorphic to a deformation of a quadric surface, and certain quotients of these varieties by $\mathbb{Z}_{2}$. They are described in detail in the Sections 2.1 and 2.3 of the present paper and called the "quadric- and $\mathbb{S}_{4}$-degenerations".

- Singular varieties arising as quotients by $\mathbb{Z}_{2}$ of a splitting Zariski $\mathbb{P}_{1^{-}}$ bundle over $Y \cong \mathbb{P}_{2}$; see Example 3.3. Abusing language, call these the "singular $\mathbb{P}_{1}$-bundles over $Y \cong \mathbb{S}_{2}$ ".

- Zariski $\mathbb{P}_{1}$-bundles over Hirzebruch-surfaces $Y$ which are constructed in Sections 3.3.1-3.3.2 by starting with a trivial $\mathbb{P}_{1}$-bundle and repeatedly performing certain elementary transformations; name these varieties the "diagonally twisted bundles".

The following is our main theorem, which is a complete classification of the 3-dimensional case. 
THEOREM 1.2. Let $X$ be a 3-dimensional complex variety which is relatively minimal over a base $Y$ and $G$ be a connected linear algebraic group acting algebraically and almost transitively on $X$ so that the kernel of $G \rightarrow \operatorname{Aut}(X)$ is discrete. Assume that $G$ is not solvable. Then,

- if $Y$ is a point, $X$ is isomorphic to $\mathbb{P}_{(1,1,2,3)}, \mathbb{P}_{(1,1,1,2)}$, or $X$ is one of the following Fano-varieties: $\mathbb{P}_{3}, \mathbb{Q}_{3}$, the 3 -dimensional quadric, $V_{5}$ or $V_{22}^{S}$.

- if $\operatorname{dim} Y=1, X$ is one of the quadric-and $\mathbb{S}_{4}$-degenerations, or a Zariski $\mathbb{P}_{2}$-bundle over $\mathbb{P}_{1}$.

- if $\operatorname{dim} Y=2$ and if $Y$ is singular, $X$ is a singular $\mathbb{P}_{1}$-bundle over $\mathbb{S}_{2}$. Otherwise, $X \cong Y \times \mathbb{P}_{1}$, where $Y$ is an arbitrary $G$-quasihomogeneous surface, or $X$ is smooth and one of the following holds:

- $X$ is one of the diagonally twisted bundles, or a splitting Zariski bundle and $Y$ is a Hirzebruch-surface $\Sigma_{n}$.

- $X$ is the full flag variety $F_{(1,2)}(3)$, a splitting Zariski bundle, a quotient of one of the relatively minimal varieties over $\Sigma_{0}$ or a blow-down of the diagonally twisted bundle $X_{\Sigma_{1}, k_{0}, 0}$. In all these cases $Y \cong \mathbb{P}_{2}$.

Although we found it easier to use the dimension of $Y$ to structure the present paper, for the reader who has been concerned with actions of semisimple groups it might be worth while to briefly discuss the classification based on the dimension of the generic orbits of a maximal semi-simple subgroup $S$ of $G$.

If $S$ acts almost transitively, the case of primary interest is that where $S \cong S L_{2}$. Here $X$ must in fact be smooth (see Lemma 4.3). In this setting the case that $\operatorname{dim} Y=0$ has been treated in the literature ([MU83], and the papers of Iskovskih). In the other cases where $\operatorname{dim} Y=1$ or 2 , one could apply the methods and results of [LV83] and [MJ90] if one would extend this to all possible isotropy groups. We choose a different approach and construct all varieties explicitly.

If the generic $S$-orbit is 2 -dimensional, the relatively minimal varieties over surfaces can be easily described. If $Y$ is a curve, we again give an explicit construction of all the possible varieties - locally these are the well-known deformations of the cones over rational normal curves of degree 
2 or 4 . The remaining case where $\operatorname{dim} Y=0$ is slightly more involved and requires a line of argumentation that does not fit well into the present work. Thus, we have chosen to treat this case in a different paper [Keb99].

Finally, if the generic $S$-orbit is 1-dimensional, then $X$ is a product $Y \times \mathbb{P}_{1}$.

The author would like to thank A. Huckleberry and T. Peternell for support and valuable discussions, and the referee for a number of suggestions improving the quality of the present paper. Part of the work on this paper was carried out during our visit to the University of Grenoble. We are thankful to the members of that institute for their kind hospitality.

\section{$\S 2$. Relatively minimal varieties over curves}

In this section we consider the following situation unless otherwise mentioned:

Assumption 2.1. Let $X$ and $G$ be as in Theorem 1.2 and $\phi: X \rightarrow Y$ be an extremal contraction to a curve.

Recall that $Y$ is necessarily normal and quasihomogeneous with respect to an algebraic action of the linear algebraic group $G$. Thus, $Y \cong \mathbb{P}_{1}$. If $X_{\eta}$ is a general $\phi$-fiber, it is del Pezzo and quasihomogeneous. Therefore it is isomorphic to either

- the projective plane $\mathbb{P}_{2}$

- $\Sigma_{0} \cong \mathbb{P}_{1} \times \mathbb{P}_{1}$

- the first Hirzebruch-surface $\Sigma_{1}$, or

- a blow-up of $\Sigma_{0}$ in at most two points $x_{1}$ and $x_{2}$ such that both natural projections $\pi_{i}: \Sigma_{0} \rightarrow \mathbb{P}_{1}$ satisfy $\pi_{i}\left(x_{1}\right) \neq \pi_{i}\left(x_{2}\right)$.

We will show that only the first two cases occur. To start with, fix some notation:

Notation 2.2. Under the above assumption, for $\eta \in Y$ let $X_{\eta}=$ $\phi^{-1}(\eta)$ be the associated fiber and $G_{\eta}$ be the stabilizer of $X_{\eta}$, i.e., the isotropy group of $\eta$.

Recall the following simple fact from minimal model theory which will be constantly used in the sequel: 
FACT 2.3. (homological equivalence of extremal curves) Let $X$ be a projective variety with $\mathbb{Q}$-factorial terminal singularities and $\phi: X \rightarrow Y$ be an extremal contraction ( $Y$ not necessarily a curve), $D \in \operatorname{Div}(X)$ an irreducible divisor and $y \in Y$ a point. If $D \cap X_{\eta}$ is a nontrivial effective divisor, then it intersects every curve in $X_{\eta}$ positively.

Proof. There is a curve $C \subset X_{\eta}$ intersecting $D$ in a finite set. So $C . D>0$. Let $C^{\prime} \subset X_{\eta}$ be any other curve. Since $\phi$ is a contraction, there exist $a, b \in \mathbb{Q}^{+}$such that $a[C]=b\left[C^{\prime}\right]$ as homology classes. Thus $D . C^{\prime}=$ $\frac{a}{b} D \cdot C>0$.

Now we can characterize the $\phi$-fibers:

LEMMA 2.4. Under Assumption 2.1, a generic fiber $X_{\eta}$ is isomorphic to $\mathbb{P}_{2}$ or to a 2-dimensional quadric.

Proof. Suppose to the contrary. Then there exist $(-1)$-curves in $X_{\eta}$. Choosing one of them, say $C$, then $D:=\overline{G . C}$ is a divisor intersecting $X_{\eta}$ in $G_{\eta} . C$, i.e., a finite number of $(-1)$-curves. We will treat the possibilities for $X_{\eta}$ separately and show that in each case the existence of $D$ yields a contradiction to the homological equivalence of extremal curves.

Assuming $X_{\eta} \cong \Sigma_{1}$, there is a unique $(-1)$-curve $C$. Hence $D \cap X_{\eta}=C$, and there are curves $C^{\prime} \subset X_{\eta}$ with $C^{\prime} . D=0$, a contradiction.

If $X_{\eta} \cong \mathbb{P}_{1} \times \mathbb{P}_{1}$ blown up in one point, there are three $(-1)$-curves $C_{1}$, $C_{2}$ and $C_{3}$ contained in $X_{\eta}$. They satisfy $C_{1} \cdot C_{2}=C_{2} \cdot C_{3}=1$ and $C_{1} \cdot C_{3}=0$. Set $D:=\overline{G \cdot C_{2}}$. Then, if $D \cap X_{\eta}$ contains $C_{1}$ and $C_{2}, C_{1} . D=0$. If $D \cap X_{\eta}$ contains $C_{2}$ and $C_{3}, C_{3} \cdot D=0$. As a last possibility, $D \cap X_{\eta}=C_{2}$. Then there exists a curve in $X_{\eta}$ which does not intersect $D$ at all. In any case, the homological equivalence of extremal curves is violated.

The last case is that $X_{\eta} \cong \mathbb{P}_{1} \times \mathbb{P}_{1}$ blown up in two points as described above. First, we remark that a 1-dimensional subgroup $H<G$ acting nontrivially on $Y$ cannot be isomorphic to $\mathbb{C}$ : if it were, since it's isotropy at a generic point $\eta \in Y$ is trivial, given any $(-1)$-curve $C \subset X_{\eta}, D:=\overline{H . C}$ would be a divisor, $D \cap X_{\eta}=C$, and there would exist curves in $X_{\eta}$ not intersecting $D$. In particular, this implies that $G$ acts as $\mathbb{C}^{*}$ on $Y$.

On the other hand, since $\operatorname{Aut}^{0}\left(X_{\eta}\right) \cong \mathbb{C}^{*} \times \mathbb{C}^{*}$, it follows that $G$ acts as a torus $\left(\mathbb{C}^{*}\right)^{3}$. A contradiction to the assumption. 


\subsection{The construction of the quadric-degenerations}

In this section we construct concrete examples of varieties over $\mathbb{P}_{1}$ which satisfy the conditions of Theorem 1.2 and whose generic fibers are smooth quadrics. For this, consider the space $V_{0}:=\mathbb{P}_{3} \times \mathbb{C}$ with coordinates $([x: y: z: w], \lambda)$. For any odd integer $k>0$, let $X_{0}^{k}$ be the quasi-projective variety given by:

$$
X_{0}^{k}:=\left\{([x: y: z: w], \lambda) \in \mathbb{P}_{3} \times \mathbb{C}: 4 x z-y^{2}=\lambda^{k} w^{2}\right\}
$$

In order to define an action of $S L_{2}$ on $X_{0}^{k}$, let $V=V_{1} \oplus V_{3}$ be the direct sum of the one- and the three-dimensional irreducible $S L_{2}$-representation spaces. After suitable choice of coordinates, the induced action on $\mathbb{P}_{3}=$ $\mathbb{P}(V)$ stabilizes all quadrics of the form $\left\{4 x z-y^{2}=\lambda w^{2}\right\}$, where $\lambda \in \mathbb{C}$ is any number. Thus, the trivial extension of this action to $\mathbb{P}_{3} \times \mathbb{C}$ yields action on $X_{0}^{k}$.

Finally, let the group $H^{*} \cong \mathbb{C}^{*}$ act as follows:

$$
\xi([x: y: z: w], \lambda)=\left(\left[x: y: z: \xi^{-k} w\right], \xi^{2} \lambda\right) .
$$

A direct calculation shows that $G:=H^{*} \times S L_{2}$ acts and stabilizes $X_{0}^{k}$.

Choosing another odd number $l$, we construct a similar quasi-projective variety $X_{\infty}^{l}$ over $\mathbb{C}$ : Again $V_{\infty}:=\mathbb{P}_{3} \times \mathbb{C}$ and $X_{\infty}^{l}:=\left\{4 x z-y^{2}=\lambda^{l} w^{2}\right\}$. Let $S L_{2}$ act as above and let $H^{*}$ act by:

$$
\xi:([x: y: z: w], \lambda) \longmapsto\left(\left[x: y: z: \xi^{l} w\right], \xi^{-2} \lambda\right) .
$$

The last step of the construction consists in gluing $V_{0}$ and $V_{\infty}$ in order to obtain a $\mathbb{P}_{3}$-bundle over $\mathbb{P}_{1}$ which contains the desired quasihomogeneous space. Define the equivalence relation

$$
\begin{aligned}
& V_{0} \ni\left(\left[x_{0}: y_{0}: z_{0}: w_{0}\right], \lambda_{0}\right) \sim\left(\left[x_{\infty}: y_{\infty}: z_{\infty}: w_{\infty}\right], \lambda_{\infty}\right) \in V_{\infty} \\
& : \Longleftrightarrow \lambda_{0} \lambda_{\infty}=1 \text { and }\left[x_{0}: y_{0}: z_{0}: w_{0}\right]=\left[x_{\infty}: y_{\infty}: z_{\infty}: w_{\infty} \lambda_{\infty}^{(k+l) / 2}\right] .
\end{aligned}
$$

Consider the equation defining $X_{0}^{k}$ and substitute the equivalent coordinates of $V_{\infty}$ :

$$
\begin{aligned}
& 4 x_{0} z_{0}-y_{0}^{2}=\lambda_{0}^{k} w_{0}^{2} \\
& \Longleftrightarrow 4 x_{\infty} z_{\infty}-y_{\infty}^{2}=\frac{1}{\lambda_{\infty}^{k}}\left(w_{\infty} \lambda_{\infty}^{(k+l) / 2}\right)^{2} \\
& \Longleftrightarrow 4 x_{\infty} z_{\infty}^{2}-y_{\infty}^{2}=w_{\infty}^{2} \lambda_{\infty}^{l}
\end{aligned}
$$

the last equation is that which defines $X_{\infty}^{l}$.

There are several things to show: 


\subsection{1. $X^{(k, l)}$ has $\mathbb{Q}$-factorial terminal singularities}

As a first step, we claim that $H^{*}$ acts trivially on the divisor class group $C l\left(X_{0}^{k}\right)$. For this, note that if $\tilde{X}^{(k, l)}$ is an $H^{*}$-equivariant resolution of the singularities, then there exists an $H^{*}$-equivariant surjection $C l\left(X^{(k, l)}\right) \rightarrow$ $C l\left(X_{0}^{k}\right)$ and an $H^{*}$-equivariant injection $C l\left(X^{(k, l)}\right) \rightarrow C l\left(\tilde{X}^{(k, l)}\right)=\operatorname{Pic}\left(\tilde{X}^{(k, l)}\right)$. But every component of $\operatorname{Pic}\left(\tilde{X}^{(k, l)}\right)$ is a compact torus, i.e., the only algebraic $H^{*}$-action is trivial — see [Mum66, Lect. 19ff] for the fact that the action of $H^{*}$ on $\operatorname{Pic}\left(\tilde{X}^{(k, l)}\right)$ is algebraic.

Second, observe that $X_{0}^{k}$ has an isolated cDV singularity at ([0 : 0 : $0: 1], 0)$, which is terminal of index one (cf. [Rei83, Par. 1]). Furthermore, $X_{0}^{1}$ is smooth.

We claim that all divisors $D \subset X_{k}$ are $\mathbb{Q}$-Cartier. Define a map $\gamma$ : $X_{0}^{k} \rightarrow X_{0}^{1}$ by $\gamma:([x, y, z, w], \lambda) \mapsto\left([x, y, z, w], \lambda^{k}\right)$. This is a quotient of $X_{0}^{k}$ by an action of $\mathbb{Z}_{k}$. Observe that

$$
D^{\prime}:=\sum_{\xi \in H^{*}, \xi^{2 k}=1} \xi D
$$

is $\mathbb{Z}_{k^{-}}$-invariant, hence Cartier. This is the place where we need " $k$ odd". As $H^{*}$ acts trivially on the divisor class group of $X_{0}^{k}, D^{\prime}$ is linearly equivalent to a multiple of $D$. Consequently, $D$ is $\mathbb{Q}$-Cartier indeed.

The same argumentation holds for $X_{\infty}^{l}$.

\subsection{2. $X^{(k, l)}$ is $G$-quasihomogeneous}

In order to see that the group actions on the quasi-projective pieces extend to the entire variety, we show that if $v_{0}=\left(\left[x_{0}: y_{0}: z_{0}: w_{0}\right], \lambda_{0}\right) \sim$ $\left(\left[x_{\infty}: y_{\infty}: z_{\infty}: w_{\infty}\right], \lambda_{\infty}\right)=v_{\infty}$ and $g \in G$, then $g . v_{0} \sim g . v_{\infty}$.

A simple calculation shows that this holds if $g \in S L_{2}$. Similarly, if $\xi \in H^{*}$

$$
\begin{aligned}
\xi\left(\left[x_{0}: y_{0}: z_{0}: w_{0}\right], \lambda_{0}\right) & =\left(\left[x_{0}: y_{0}: z_{0}: \xi^{-k} w_{0}\right], \xi^{2} \lambda_{0}\right) \\
\xi\left(\left[x_{\infty}: y_{\infty}: z_{\infty}: w_{\infty}\right], \lambda_{\infty}\right) & =\left(\left[x_{\infty}: y_{\infty}: z_{\infty}: \xi^{l} w_{\infty}\right], \xi^{-2} \lambda_{\infty}\right)
\end{aligned}
$$

Now note that $\left(\lambda_{0} \xi^{2}\right)\left(\lambda_{\infty} \xi^{-2}\right)=\lambda_{0} \lambda_{\infty}$ and $\xi^{l} w_{\infty}\left(\lambda_{\infty} \xi^{-2}\right)^{(k+l) / 2}=w_{0} \xi^{-k}$, showing that $\xi\left(\left[x_{0}: y_{0}: z_{0}: w_{0}\right], \lambda_{0}\right) \sim \xi\left(\left[x_{\infty}: y_{\infty}: z_{\infty}: w_{\infty}\right], \lambda_{\infty}\right)$. Due to the product structure, $g . v_{0} \sim g \cdot v_{\infty}$ for all $g \in G$.

2.1.3. There exists an extremal ray contraction $X^{(k, l)} \rightarrow \mathbb{P}_{1}$

Perform a relative Mori contraction $\psi: X \rightarrow Z$ over $\mathbb{P}_{1}$. Note that if $X_{\mu}$ is an arbitrary fiber of the map $X \rightarrow \mathbb{P}_{1}$, then all curves contained in 
$X_{\mu}$ are equivalent as homology cycles: this is clear for the singular fibers over 0 and $\infty$ because they are singular quadrics, and also true for the generic fibers because the action of $\pm 1 \in H^{*}$ swaps horizontal and vertical directions. Consequently, $\psi(X)=\mathbb{P}_{1}$, and the claim is shown.

\subsection{The Characterization of Quadric-Degenerations}

We will show that every relatively minimal variety over $\mathbb{P}_{1}$ whose generic fiber $X_{\eta}$ is a quadric is isomorphic to some $X^{(k, l)}$. Identify $X_{\eta}$ with $\mathbb{P}_{1} \times \mathbb{P}_{1}$, and let $\pi_{1}$ and $\pi_{2}: X_{\eta} \rightarrow \mathbb{P}_{1}$ be the standard projections. Call $\pi_{1}$-fibers "vertical" and $\pi_{2}$-fibers "horizontal".

Proposition 2.5. (Characterization of Quadric-Degenerations) Under Assumption 2.1, if the generic fiber $X_{\eta}$ is isomorphic to a 2-dimensional quadric, then $X$ is isomorphic to one of the quadric-degenerations constructed in Section 2.1.

We subdivide the proof into a number of steps:

Step 1: Description of the $S$-Action. Let $C \subset X_{\eta}$ be a horizontal curve and $H<G$ a one-parameter group acting non-trivially on $Y$. Let $H_{\eta}<H$ be the stabilizer of $X_{\eta}$ and set $E:=\overline{H . C}$. If $E_{\eta}:=E \cap X_{\eta}=H_{\eta} . C$ is a union of finitely many horizontal curves, then $E_{\eta}$ does not intersect a general horizontal curve, contradicting the homological equivalence of extremal curves (see Fact 2.3). Thus there exists $h \in H_{\eta}: h . C$ is not horizontal. In particular, $H_{\eta}$ is not trivial and $H \cong \mathbb{C}^{*}$ is a torus.

Let $S$ be a maximal semi-simple subgroup of $G$. As all one-parameter subgroups of $G$ acting non-trivially on the base are necessarily tori, $S$ acts trivially on $Y$. If some $S^{\prime} \cong S L_{2}$ in $S$ would act only on one factor of $X_{\eta} \cong \mathbb{P}_{1} \times \mathbb{P}_{1}$, we derive a contradiction as follows: let $T<S^{\prime}$ be a maximal torus and $F \subset X$ it's fixed point set. Since $X_{\eta}$ was chosen to be a general $\phi$-fiber, the $S^{\prime}$-orbits in the neighboring fibers are 1-dimensional, too. So $F$ is a divisor. By assumption, $F \cap X_{\eta}$ is the union of two horizontal (or vertical) curves, a contradiction to the homological equivalence of extremal curves. Thus $S=S L_{2}$ and it's action is diagonal. In particular, there exists an $S$-invariant diagonal $\delta \subset X_{\eta}$.

Step 2: The Embedding into a Zariski Bundle. We claim $\delta$ is also invariant under $G_{\eta}$. Assume to the contrary and let $g \in G_{\eta}$ be an element not stabilizing $\delta$. But $S$ has only two orbits in $X_{\eta}$, namely $\delta$ and $X_{\eta} \backslash \delta$, 
so that any group containing $S$ and $g$ acts transitively on $X_{\eta}$, i.e., contains $S L_{2} \times S L_{2}$. But this is absurd, as we have seen.

Set $D:=\overline{H . \delta}$. The desingularization $\tilde{D}$ of $D$ is then quasihomogeneous. By classification, $\tilde{D} \cong \mathbb{P}_{1} \times \mathbb{P}_{1}$. This has two consequences: First, as $S$ acts transitively on the fibers of $\tilde{D} \rightarrow Y$, all fibers of the map $\tilde{D} \rightarrow D$ are discrete, and the $S$-action on $D$ does not have a fixed point. Since the singularities of $X$ are isolated, $D$ does not meet the singular set of $X$. Thus, $D$ is Cartier. Second, if $U<S$ is a unipotent group, then the $U$-fixed points in $D$ form a curve which is mapped injectively onto $\mathbb{P}_{1}$. This already shows that $\phi$ has maximal rank along these curves so that there are no multiple fibers. Furthermore, is $X_{\nu}$ is any $\phi$-fiber, then $X_{\nu}$ is smooth along $D \cap X_{\nu}$.

Recall that $\phi$ being an extremal contraction implies that $D$ is relatively ample. As $X_{\nu} \cap D$ is ample and $S$-invariant, there is no $S$-invariant curve in $X_{\nu} \cap D$. In particular, the singular set of $X_{\nu}$ is discrete. Since all fibers are Cohen-Macaulay, it follows from Serre's criterion that they are normal. But the only normal $S L_{2}$-surfaces containing an ample $S$-invariant divisor of self-intersection 2 are the 2-dimensional quadrics. Thus, we conclude that the dimension of the linear system $\left|X_{\nu} \cap D\right|$ is independent of $\nu \in Y$ and that $D$ is relatively very ample, i.e., there exists an embedding $X \rightarrow \mathbb{P}(E)$, where $E:=\phi_{*}(\mathcal{O}(D))$ is a rank 4 -vector bundle.

Step 3: Local Description. Knowing that the intersection of $D \cap X_{\eta}$ yields an equivariant embedding $X_{\eta} \rightarrow \mathbb{P}_{3}$, one sees that there is an $S$-stable splitting $E=E_{3} \oplus E_{1}$, where $E_{3}$ is of rank three and $S$ acts on the fibers via it's irreducible 3-dimensional representation and $E_{1}$ is 1-dimensional with trivial $S$-action. Let $T<S L_{2}$ be the diagonal matrices. Then the direct sum decomposition of the irreducible $S L_{2}$-representations into $T$-weight spaces yields a $T$-stable splitting $E_{3}=E_{3}^{-2} \oplus E_{3}^{0} \oplus E_{3}^{2}$, where $T$ acts on the total space of $E_{3}^{i}$ with weight $i$.

As a next step, choose a $G$-invariant affine subset $\mathbb{C} \cong U^{0} \subset Y \cong \mathbb{P}_{1}$ containing one of the $G$-fixed points in $Y$. Let $y$ be a bundle coordinate for $E_{3}^{0}$ over $U^{0}$; we view that as giving a $T$-equivariant map from $E_{3}^{0}$ into the standard 3-dimensional $S L_{2}$-representation space $V_{2}$. In order to obtain an $S L_{2}$-equivariant map $\left.E_{3}\right|_{U^{0}} \rightarrow V_{2}$, conjugate $y$ with the going-up and going-down operators in $S L_{2}$. This way we obtain coordinates $x$ and $z$ for $E_{3}^{-2}$ and $E_{3}^{2}$, respectively, giving the desired map to $V_{2}$.

Use these coordinates to view $X^{0}:=\phi^{-1}\left(U^{0}\right)$ as a subset of $\mathbb{P}_{3} \times \mathbb{C}$. The generic fiber is an $S$-invariant quadric, hence given by $c\left(4 x z-y^{2}\right)=c^{\prime} w^{2}$ 
where $c, c^{\prime} \in \mathbb{C}^{*}$. Thus, after appropriate choice of coordinates, $X \cap \phi^{-1}\left(U_{0}\right)$ is given by $4 x z-y^{2}=\lambda^{k} w^{2}$ or $\lambda^{k}\left(4 x z-y^{2}\right)=w^{2}$ with $k \geq 0$. The latter case is excluded, because all $\phi$-fibers are reduced. Furthermore, if $k$ is even, the closure of $D^{\prime}:=\{x=0\} \cap \phi^{-1}\left(U_{0}\right)$ is a divisor intersecting the generic fiber in a fiber of the ruling: a contradiction to the homological equivalence of extremal curves (see Fact 2.3 or to $D^{\prime}$ being $\mathbb{Q}$-Cartier. The remaining case occurs indeed, as was shown in Section 2.1.

Step 4: End of the Proof. After a similar argumentation for the part of $X$ over $U_{\infty}=\mathbb{P}_{1} \backslash\{0\}$, we again obtain the equations of one of the quadric-degenerations described in Section 2.1. Note that the transition map must commute with the action of $S L_{2}$. On the other hand, the only automorphisms of the smooth quadric commuting with the diagonal action of $S L_{2}$ are the identity and the involution which interchanges the horizontal and vertical directions. But $H^{1}\left(\mathbb{P}_{1}, \mathbb{Z}_{2}\right)=0$, so that either choice gives a variety which is isomorphic to one of the examples.

\subsection{The Construction of the $\mathbb{S}_{4}$-Degenerations}

Now we consider the case where $X_{\eta} \cong \mathbb{P}_{2}$. In analogy with the construction of the quadric degenerations, set $V_{0}:=\mathbb{P}_{5} \times \mathbb{C}$ with coordinates ( $[a: b: c: e: f: g], \lambda)$ and let $S L_{2}$ act on $V_{0}$ via it's 5-dimensional irreducible representation on $a \ldots f$. For a given $k \in \mathbb{N}$, let the group $H^{*} \cong \mathbb{C}^{*}$ act on $V_{0}$ by

$$
\xi:([a: b: c: e: f: g], \lambda) \longmapsto\left(\left[a: b: c: e: f: \xi^{-2 k} g\right], \xi^{2} \lambda\right) .
$$

and define $X_{0, q}^{k}$ to be the variety given by the ideal

$$
\begin{array}{ll}
3 e^{2}-8 c f+4 f \lambda^{k} g, & c e-6 b f+e \lambda^{k} g, \\
3 b e-48 a f+2 c \lambda^{k} g+2\left(\lambda^{k} g\right)^{2}, & c^{2}-36 a f+2 c \lambda^{k} g+\left(\lambda^{k} g\right)^{2}, \\
b c-6 a e+b \lambda^{k} g, & 3 b^{2}-8 a c+4 a \lambda^{k} g .
\end{array}
$$

Note that for a given $\lambda \in \mathbb{C}^{*} \subset \mathbb{P}_{1}$, the fiber $X_{\lambda}$ is isomorphic to $\mathbb{P}_{2}$; the embedding is given by $[x: y: z] \rightarrow\left(\left[x^{2}: 2 x y: 2 x z+y^{2}: 2 y z: z^{2}:\right.\right.$ $\left.\left.\lambda^{-k}\left(4 x z-y^{2}\right)\right], \lambda\right)$.

Given another number $l \in \mathbb{N}$, construct $X_{\infty, q}^{l} \subset V_{\infty}=\mathbb{P}_{5} \times \mathbb{C}$ with $H^{*}$ action given by $\xi:([a: b: c: e: f: g], \lambda) \rightarrow\left(\left[a: b: c: e: f: \xi^{2 l} g\right], \xi^{-2} \lambda\right)$. The same calculations as in Section 2.1 show that $X_{0, q}^{k}$ and $X_{\infty, q}^{l}$ glue 
together to a variety $X^{(k, l, q)}$ via the relation

$$
\begin{aligned}
& \left(\left[a_{0}: b_{0}: c_{0}: e_{0}: f_{0}: g_{0}\right], \lambda_{0}\right) \sim\left(\left[a_{\infty}: b_{\infty}: c_{\infty}: e_{\infty}: f_{\infty}: g_{\infty}\right], \lambda_{\infty}\right) \\
& : \Longleftrightarrow \lambda_{0} \lambda_{\infty}=1 \text { and } \\
& \quad\left[a_{0}: b_{0}: c_{0}: e_{0}: f_{0}: g_{0}\right]=\left[a_{\infty}: b_{\infty}: c_{\infty}: e_{\infty}: f_{\infty}: g_{\infty} \lambda_{\infty}^{k+l}\right] .
\end{aligned}
$$

It is still to be shown that $X^{(k, l, q)}$ has $\mathbb{Q}$-factorial terminal singularities and it suffices to show this for $X_{0, q}^{k}$. Define $X_{0}^{k}$ as in Section 2.1, even if $k$ is not odd. Let $\mathbb{Z}_{2}$ act on $X_{0}^{k}$ by

$$
(-1):([x: y: z: w], \lambda) \longmapsto([x: y: z:-w], \lambda)
$$

We claim that $X_{0, q}^{k}$ is the quotient of $X_{0}^{k}$ by $\mathbb{Z}_{2}$. The quotient map is given by

$$
([x: y: z: w], \lambda) \longmapsto\left(\left[x^{2}: 2 x y: 2 x z+y^{2}: 2 y z: z^{2}: w^{2}\right], \lambda\right) .
$$

and a direct calculation shows that the quotient is isomorphic to $X_{0, q}^{k}$. See [Rei87, p. 391] for the fact that the singularities of the quotient are terminal. In order to show that they are $\mathbb{Q}$-factorial, it is sufficient to see that all $\mathbb{Z}_{2^{-}}$ invariant divisors in $X_{0}^{k}$ are $\mathbb{Q}$-factorial, if restricted to the quasi-projective parts. If $k$ is odd, this was shown for any divisor. If $k$ is even and $D \subset X_{0, q}^{k}$ a $\mathbb{Z}_{2}$-invariant divisor, one can argue similarly and use the fact that

$$
D^{\prime}:=\sum_{\xi \in H^{*}, \xi^{2 k}=1} \xi D
$$

is a multiple of $D$ and Cartier.

The same argumentation as in Section 2.1 shows that there exists an extremal ray contraction $X^{(k, l, q)} \rightarrow \mathbb{P}_{1}$.

\subsection{The Characterization of the $\mathbb{S}_{4}$-Degenerations}

This is in full analogy to the quadric case.

Proposition 2.6. (Characterization of the $\mathbb{S}_{4}$-Degenerations) Under the Assumption of 2.1 , if the generic $\phi$-fiber is isomorphic to $\mathbb{P}_{2}$, then $X$ is either a Zariski $\mathbb{P}_{2}$-bundle or one of the $\mathbb{S}_{4}$-degenerations constructed in Section 2.3.

Proof. If $X$ is smooth, take a one-parameter subgroup $H<G$ acting non-trivially on the base $Y$. Given a generic fiber $X_{\eta}$, there will always be a line $L \subset X_{\eta}$, invariant under the action of the isotropy group $H_{\eta}$. 
Then $D:=\overline{H . L}$ is a relatively ample divisor intersecting $X_{\eta}$ in $L$. See [Fuj85, Lem. 2.12] for the fact that this yields an embedding of $X$ into $\mathbb{P}\left(\phi_{*} \mathcal{O}(D)\right)$ which is a $\mathbb{P}_{2}$-bundle. This must be an isomorphism. Note that $X$ is automatically smooth if $S$, the semi-simple part of $G$, acts non-trivially on $Y$.

If $X$ is singular and there is a subgroup $S^{\prime}<S, S^{\prime} \cong S L_{2}$, acting trivially on $Y$ and having a fixed point on generic fibers, then the subvariety $\left\{x \in X \mid \operatorname{dim} S^{\prime} . x<2\right\}$ contains a divisor $D$ which intersects $X_{\eta}$ in an $S^{\prime}$ homogeneous line. Now argue as in the proof of Proposition 2.5. Note that, since $D$ does not contain a fixed point, it is Cartier.

It remains to consider the case where $S \cong S L_{2}$ acts trivially on $Y$ and stabilizes a quadric curve in $X_{\eta}$. As above, let $D$ be the union of these curves. In complete analogy to the proof of Proposition 2.5, all fibers are isomorphic to $\mathbb{P}_{2}$ or $\mathbb{S}_{4}, D$ is Cartier and yields an embedding into a $\mathbb{P}_{5}$-Bundle $\mathbb{P}(E)$. Here $E$ splits $S$-equivariantly into a direct sum of a 5 -dimensional bundle $E_{5}$, where $S$ acts via it's irreducible representation, and a 1-dimensional bundle $E_{1}$ where the $S$-action is trivial. Furthermore, the subbundle $\mathbb{P}\left(E_{5}\right)$ is the unique hyperplane intersecting $X$ in $D$.

We continue to argue as in 2.5 , using the fact that all $S L_{2}$-invariant subsets in $\mathbb{P}_{5}$, isomorphic to $\mathbb{P}_{2}$ and not contained in the $S L_{2}$-invariant hyperplane are given by

$$
\begin{array}{ll}
3 e^{2}-8 c f+4 f \lambda g, & c e-6 b f+e \lambda g \\
3 b e-48 a f+2 c \lambda g+2 \lambda^{2} g^{2}, & c^{2}-36 a f+2 c \lambda g+(\lambda g)^{2} \\
b c-6 a c+b \lambda g, & 3 b^{2}-8 a c+4 a \lambda g
\end{array}
$$

where $\lambda \in \mathbb{C}^{*}$. Consequently, $X$ is locally given by the equations from Section 2.3. There is no choice of how the affine parts can be $S L_{2}$-equivariantly glued.

\section{$\S$ 3. Relatively Minimal Varieties over Surfaces}

The primary aim of this section is to classify the relatively minimal varieties over surfaces. The following lemma describes the case where a semi-simple group acts in fiber direction.

LEMMA 3.1. In the situation of Theorem 1.2, let $Y$ be a surface. If $S<G$ is a semi-simple group which acts trivially on $Y$, then $X \cong Y \times \mathbb{P}_{1}$. In particular, $X$ and $Y$ are smooth. 
Note that some of our arguments involve linearization of group actions at fixed points. See [Huc90] or [HO80, p. 11f] for information about this.

Proof. Since $\phi$ is an extremal contraction and $X$ is assumed to be $\mathbb{Q}$-factorial, all fibers must be of dimension 1 .

Note that $S$ acts transitively on the generic fibers. Thus, $S \cong S L_{2}$ and $S$ has no fixed points: a linearization of the $S L_{2}$-action would give a contradiction.

Consequently, $S$ acts transitively on all fibers, and if $U<S$ is a maximal connected unipotent subgroup, and $\Sigma$ it's fixed point set, then $X=S . \Sigma \cong$ $\mathbb{P}_{1} \times \Sigma$.

Due to the preceding Lemma, we may consider for the rest of this section that the semi-simple part of $G$ acts non-trivially on $Y$ :

Assumption 3.2. Let $X$ and $G$ be as in Theorem 1.2 and let $\phi$ : $X \rightarrow Y$ be an extremal contraction to a surface. Let $S<G$ be a maximal semi-simple subgroup and assume that no simple factor of $S$ acts trivially on $Y$.

\subsection{Varieties over Singular Surfaces}

We start with the construction of the relatively minimal varieties over a singular surface.

EXAMPLE 3.3. Set $\tilde{X}:=\mathbb{P}\left(\mathcal{O}_{\mathbb{P}_{2}}(e) \oplus \mathcal{O}_{\mathbb{P}_{2}}\right)$. The automorphism group of $\tilde{X}$ contains a product $G:=S L_{2} \times \mathbb{C}^{*}$, where $S L_{2}$ has a fixed point in $\mathbb{P}_{2}, 2$-dimensional orbits in $\tilde{X}$ and acts trivially on the fiber over the fixed point. The factor $\mathbb{C}^{*}$ acts in fiber direction only, i.e., trivially on $\mathbb{P}_{2}$. Embed $\mathbb{Z}_{2}$ diagonally into $G$, i.e., consider the subgroup generated by $(\operatorname{Diag}(-1,-1),-1)$. Then $X:=\tilde{X} / \mathbb{Z}_{2}$ is a singular variety over $\mathbb{S}_{2}$, the cone over a non-singular conic in $\mathbb{P}_{2}$.

We will see that the examples constructed above are the only varieties which satisfy the assumption of the Main Theorem 1.2 and are relatively minimal over a singular surface.

Notation 3.4. Call a divisor $D \subset X$ a "rational section" iff it intersects the generic $\phi$-fiber with multiplicity 1 . Note that a rational section is a section iff it does not contain a whole $\phi$-fiber. 
Proposition 3.5. Under the Assumption 3.2, assume that $Y$ is singular. Then $X$ is one of the varieties constructed in Example 3.3 .

Proof. As a first step, construct a rational section. By assumption, $S$ acts non-trivially on $Y$. It follows from the classification that $Y \cong \mathbb{S}_{n}$, the cone over a rational normal curve and $S \cong S L_{2}$. The $S$-isotropy $S_{\eta}$ of a generic point $\eta \in Y$ is an extension of a maximal unipotent group by a cyclic group. Thus, $S_{\eta}$ fixes at least one point in the fiber $X_{\eta}$ so that the closure $E$ of at least one $S$-orbit is a rational section indeed.

The Weil-divisor $E$ is not Cartier, or else use [Fuj85, Lem. 2.12] and obtain a contradiction to " $Y$ singular". Thus, $X$ is singular.

The next step is to construct a cover of $X$. Observe that a fiber $X_{\mu}$ that intersects the singular set $\operatorname{Sing}(X)$ is pointwise $S$-fixed. Linearize the $S$-action at a generic point $f \in X_{\mu}$ and note that, after proper choice of coordinates, one may identify a neighborhood $U(f) \cong \Delta_{1} \times \Delta_{2}$, where $\Delta_{1}$ is a one-dimensional and $\Delta_{2}$ a 2-dimensional ball. We can assume that $S$ acts only on the second component and that the map $\left.\phi\right|_{U(f)}$ is given by the projection to the second factor followed by taking the quotient by $\mathbb{Z}_{n}$.

Let $\gamma: \mathbb{P}_{2} \rightarrow \mathbb{S}_{n}$ be the natural cyclic cover. Observe that $\gamma$ is $S$ equivariant and set $X^{\prime}:=X \times_{\mathbb{S}_{n}} \mathbb{P}_{2}$. Calculating the preimage of $U(f)$ one obtains $\Delta_{1} \times\left(n\right.$ copies of $\Delta_{2} \subset \mathbb{C}^{4}$ meeting in a point $)$. If $\tilde{X}$ is the normalization of $X^{\prime}$, the preimage of $U(f)$ becomes $\Delta_{1} \times\left(\Delta_{2} \coprod \cdots \coprod \Delta_{2}\right)$, so that $\tilde{X}$ is a $n: 1$ cover over $X$, with finite singular set. The calculation also shows that $\tilde{X}$ is Galois with group $\Gamma=\mathbb{Z}_{n}$.

We claim that $\tilde{X}$ is a split Zariski $\mathbb{P}_{1}$-bundle over $\mathbb{P}_{2}: \tilde{X} \cong \mathbb{P}(\mathcal{L} \oplus \mathcal{O})$. If $\tilde{\phi}: \tilde{X} \rightarrow \mathbb{P}_{2}$ is the natural map, consider the $\tilde{\phi}$-fiber $\tilde{X}_{\mu}$ over the unique $S$-fixed point in $\mathbb{P}_{2}$. As it's image in $X$ is pointwise $S$-fixed, $\tilde{X}_{\mu}$ is, too. Using the linearization argument, let $\tilde{E}$ be the closure of a generic $S$-orbit, intersecting $\tilde{X}_{\mu}$ in a generic point. Observe that $\tilde{E}$ contains a unique $S$-fixed point and is smooth there. Consequently, $\tilde{E}$ is a smooth section, $\tilde{X}=\mathbb{P}(\mathcal{E})$ is a Zariski $\mathbb{P}_{1}$-bundle and, as all Ext-groups on $\mathbb{P}_{2}$ vanish, $\mathcal{E}$ is split: we may assume $\mathcal{E}=\mathcal{O}_{\mathbb{P}_{2}}(e) \oplus \mathcal{O}_{\mathbb{P}_{2}}$.

We must show that the action of $\Gamma$ is the same as in the Example 3.3 above. Identify an $S L_{2}$-invariant neighborhood of $\tilde{X}_{\mu}$ with $\mathbb{C}^{2} \times \mathbb{P}_{1}$ in a way that $S$ acts on the first factor only. By equivariance, $\Gamma$ maps $S$-orbits to $S$ orbits. Consequently, the quotient by $\Gamma$ has two cyclic quotient singularities of type $\frac{1}{n}(1,1, a)$ and $\frac{1}{n}(1,1,-a)$. As quotient singularities are terminal only 
if of type $\frac{1}{n}(1, a,-a)$ (cf. [Rei87, Sect. 5.3]), $n=2$ and $a=1$. This yields the claim.

\subsection{Varieties over Smooth Surfaces}

In [Keb98] we were discussing the possibility to compactify homogeneous spaces to particularly simple varieties. We refer the reader to Section 5.2 of that paper for a proof of the fact that the $X$ is a automatically a Zariski $\mathbb{P}_{1}$-bundle if $X$ is relatively minimal over a smooth surface and $G$ is not solvable. The rest of Section 3 is concerned with an investigation which rank-two vector bundles do actually occur. We assume that $X$ is a Zariski bundle without further mention.

Remark that under the Assumption 3.2 $Y$ is a rational $G$-quasihomogeneous surface with non-trivial $S$-action. Since $X$ is now supposed to be smooth, $Y$ is smooth, so that $Y \cong \Sigma_{n}$ or $\mathbb{P}_{2}$. Later on, we will consider these cases separately.

Notation 3.6. Let $\phi: X \rightarrow Y$ be as above and assume that there exists a map $\pi: Y \rightarrow Z \cong \mathbb{P}_{1}$, e.g. if $Y$ is isomorphic to a (blown-up) Hirzebruch surface $\Sigma_{n}$. Then, if $F \in Z$ is a generic point, set $F_{Y}:=\pi^{-1}(F)$ and $F_{X}:=\phi^{-1}\left(F_{Y}\right)$.

\subsection{The Construction of the Diagonally Twisted Bundles}

The following varieties will be of great importance in the classification:

\subsubsection{The Construction of the $X_{\Sigma_{n}, k_{0}, k_{\infty}}$}

Let $Y$ be the Hirzebruch-surface $\Sigma_{n}, n>0$ and $X:=Y \times \mathbb{P}_{1}$. Let $S:=S L_{2}$ act on $Y$ and $\mathbb{P}_{1}$ and let $S$ act on $X$ diagonally, i.e., simultaneously on both components.

We claim that $S$ acts almost transitively on $X$ and that the exceptional set (i.e., the complement of the open orbit) contains a unique $S$-invariant section over $Y$. In order to see this, let $B<S$ be the Borel group of $S$ stabilizing $F_{X}$. The $B$-action on $F_{X}$ is very special: Since the $S$-action on $\Sigma_{n}$ stabilizes the 0 - and the $\infty$-sections, the $B$-action on $F_{X}$ stabilizes two fibers. Therefore the unipotent part $B_{U}$ of $B$ acts in fiber direction only, showing that the $B$-action on $F_{X}$ is quasihomogeneous and that there is exactly one $B$-invariant section in $F_{X}$. Using the $S$-action in order to move $F_{X}$ around shows that $S$ does indeed act almost transitively and that there is a unique $S$-invariant rational section $E$. The fact that $S$ does not have 
any fixed points on $Y$ immediately implies that $E$ is indeed a section and $E \cong Y \cong \Sigma_{n}$. Let $E_{0}$ and $E_{\infty}$ denote the 0 - and $\infty$-section of $E$, respectively.

The curves $E_{0}$ and $E_{\infty}$ are the only $S$-invariant subsets in $E$. We can now perform an elementary transformation with center being $E_{0}$ or $E_{\infty}$, obtaining a new $\mathbb{P}_{1}$-bundle which is not necessarily the compactification of a line bundle. By elementary transformation we understand the process of blowing up $E_{0}$ and then blowing down the strict transform of $\phi^{-1} \phi\left(E_{0}\right)$. Such transformations always exist; see [Mar73] for a complete reference. Since the centers of the transformations are $S L_{2}$-invariant, $S L_{2}$ acts on the transformed varieties, and the entire procedure is equivariant.

The strict transform of $E$ is again invariant and isomorphic to $\Sigma_{n}$ so that one may iterate the process. Let $X_{\Sigma_{n}, k_{0}, k_{\infty}}$ be the variety obtained by transforming $k_{0}$ times with center being the 0 - and $k_{\infty}$ times with center being the $\infty$-section of $E$. Let $F_{k_{0}, k_{\infty}}$ be the strict transform of $F_{X}$ in $X_{\Sigma_{n}, k_{0}, k_{\infty}}$.

As above, $B_{U}$ acts on $F_{k_{0}, k_{\infty}}$ by adding multiples of a section. Note that $F_{k_{0}, k_{\infty}} \cong \Sigma_{k_{0}+k_{\infty}}$ and the sections added by $B_{U}$ vanish of order $k_{0}$ at $F_{k_{0}, k_{\infty}} \cap E_{0}$ and of order $k_{\infty}$ at $F_{k_{0}, k_{\infty}} \cap E_{\infty}$.

3.3.2. The Construction of the $X_{\Sigma_{0}, n}$

Let $S:=S L_{2}$ act diagonally on $Y=\Sigma_{0}$. Since $S$ is a simply-connected semi-simple group and $H^{1}(Y, \mathcal{O})=0$, the $S$-action on $Y$ can be lifted to the total space of any line bundle $\mathcal{O}(n, m)$ over $Y$; see [HO80, p. 98] for details. For $n \in \mathbb{N}^{+}$the group $S$ therefore acts on the compactification $X=\mathbb{P}(\mathcal{O}(n,-n) \oplus \mathcal{O})$ which is a $\mathbb{P}_{1}$-bundle $\phi: X \rightarrow Y$. This lifting is unique up to the $\mathbb{C}^{*}$-action given by the principal $\mathbb{C}^{*}$-actions on the first factor.

Let $\sigma_{0}$ and $\sigma_{\infty}$ be the $S$-invariant sections defined by the direct sum structure. Since there are no other sections, it follows that $S$ acts transitively on the complement $X \backslash\left(\sigma_{0} \cup \sigma_{\infty} \cup \Delta_{X}\right)$, where $\Delta_{X}$ is the preimage $\pi^{-1}(\Delta)$ of the $S$-invariant diagonal $\Delta$ in $Y$.

If $i: \Delta \hookrightarrow Y$ is the canonical embedding, then $i^{*}(\mathcal{O}(n,-n))$ is trivial. Thus $\left.\phi\right|_{\Delta_{X}}: \Delta_{X} \rightarrow Y$ is the trivial $\mathbb{P}_{1}$-bundle and therefore all $S$-orbits in $\Delta_{X}$ are 1-dimensional sections over $\Delta=\mathbb{P}_{1}$.

Let $C=S x$ be such a section which does not lie in $\sigma_{0} \cup \sigma_{\infty}$ and define $X_{\Sigma_{0}, n}$ to be the elementary transformation of $X$ with respect to $C$ in $\Delta_{X}$. This manifold is still an $S$-equivariant $\mathbb{P}_{1}$-bundle over $Y$. However now the transforms $\sigma_{0}^{\prime}$ and $\sigma_{\infty}^{\prime}$ intersect transversally in an $S$-orbit $C^{\prime}=S x \cong \mathbb{P}_{1}$ 
over $\Delta$.

Given any two $S$-orbits $C_{i}:=S x_{i}$ as above, there exists a unique transformation $g$ of the $\mathbb{C}^{*}$-action which commutes with the $S$-action so that $g\left(C_{1}\right)=C_{2}$. This defines an $S$-equivariant isomorphism between the spaces $X_{\Sigma_{0}, n}^{1}$ and $X_{\Sigma_{0}, n}^{2}$ which are defined by elementary transformations along $C_{1}$ and $C_{2}$ respectively. In this sense the diagonally twisted bundle $X_{\Sigma_{0}, n}$ is uniquely defined.

\subsection{The Classification of $S$-quasihomogeneous Bundles}

3.4.1. Bundles over $\Sigma_{n}$

The following lemma gives a first characterization of split Zariski bundles:

LEMMA 3.7. Under the Assumption 3.2, assume additionally that $Y \cong$ $\Sigma_{n}$ and that $F_{X} \cong \mathbb{P}_{1} \times \mathbb{P}_{1}$, where $F_{X}$ is defined as in Notation 3.6. Then $X$ is isomorphic to a fibered product: $X \cong Y{ }_{Z} Y^{\prime}$. In particular, $X$ is a split Zariski bundle: $X \cong \mathbb{P}(\mathcal{L} \oplus \mathcal{O})$.

Proof. The space $X$ has relative Picard-number 2 over $Z$ and the general fiber $F_{X}$ is Fano. Thus, there exists a second Mori-contraction $\phi^{\prime}$ : $X \rightarrow Y^{\prime}$ over $Z$ which is different from $\phi$.

The Picard-number of $Y^{\prime}$ is 2 , so $Y^{\prime}$ is not a curve. If $\operatorname{dim} Y^{\prime}=3$, then the contraction was divisorial inducing a contraction from $F_{X}$ to a surface, which is obviously impossible. Therefore, the contraction $\phi^{\prime}$ is of fiber type. Consequently $X$ is a $\mathbb{P}_{1}$-bundle over $Y^{\prime}$ with fibers being the horizontal curves in $F_{X}$ and their translates.

With the aid of the preceding lemma we can now carry out the classification of $S$-quasihomogeneous bundles over $\Sigma_{n}$.

Proposition 3.8. (Characterization of $\left.X_{\Sigma_{n}, k_{0}, k_{\infty}}\right)$ Under the Assumption 3.2, if $Y \cong \Sigma_{n}, n>0$ and $S$ acts almost transitively on $X$, then either $X \cong Y \times \mathbb{P}_{1}$, or $S \cong S L_{2}$ and there exist numbers $k_{0}, k_{\infty} \geq 0$ such that $X \cong X_{\Sigma_{n}, k_{0}, k_{\infty}}$ is one of the diagonally twisted bundles constructed in Section 3.3.1.

Proof. Since no factor of $S$ acts trivially on $Y, S \cong S L_{2}$. There are exactly two $S$-invariant curves $\sigma_{0}, \sigma_{\infty}$ in $Y$; these are sections over $Z$. Call the preimages $\phi^{-1}\left(\sigma_{0}\right)$ and $\phi^{-1}\left(\sigma_{\infty}\right)$ of these sections $A_{0}$ and $A_{\infty}$, respectively. 


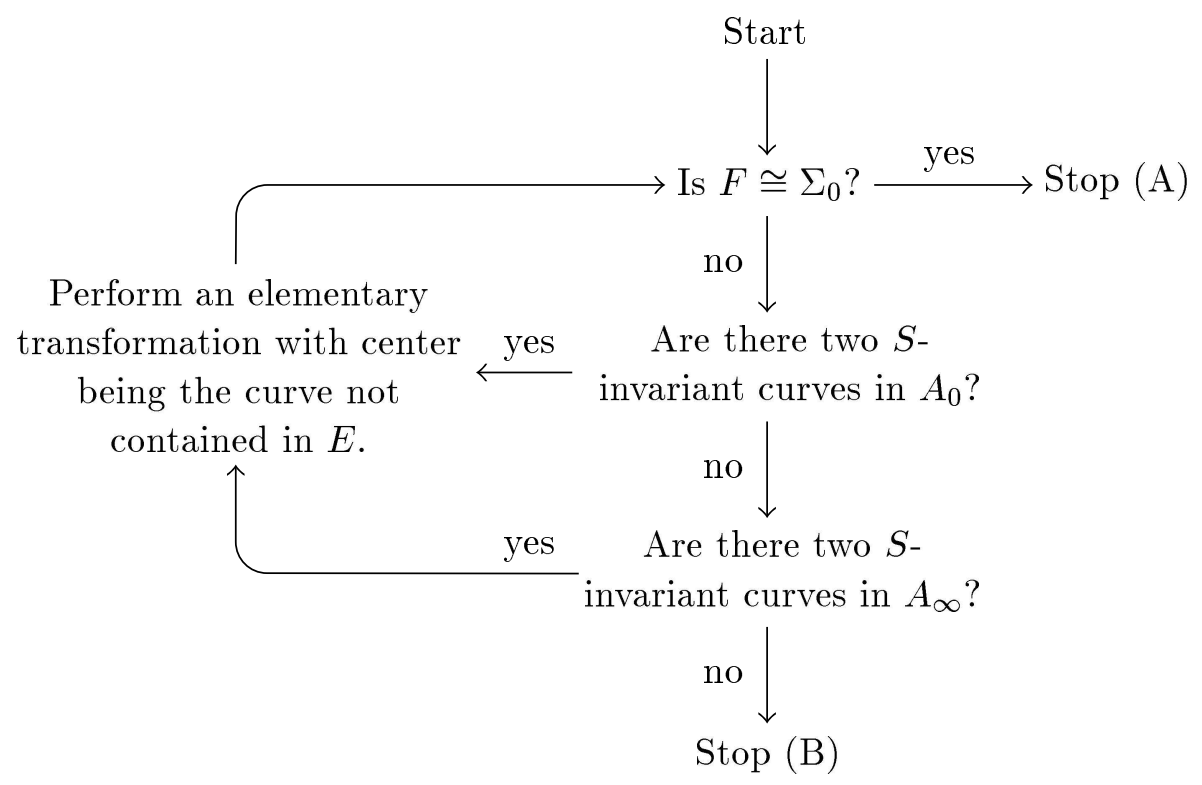

Figure 1: an algorithm for simplifying special $\mathbb{P}_{1}$-bundles

The $S$-invariant divisors in $X$ : Let $F_{X}$ be as in Notation 3.6. If $B$ is the Borel group in $S L_{2}$ stabilizing $F_{X}$, then, because $F_{X} \cap A_{0}$ and $F_{X} \cap A_{\infty}$ are invariant, $B_{U}$, the unipotent part of $B$, acts trivially on the base. Instead, $B_{U}$ acts on the fibers of $\left.\phi\right|_{F_{X}}$ and fixes a unique point in each. Consequently there exists a unique $B$-invariant section in $F_{X}$; other $B$-invariant curves are the fibers $A_{0} \cap F_{X}$ and $A_{\infty} \cap F_{X}$. Using $S$ to move $F_{X}$, one sees that the only closed $S$-invariant divisors are $A_{0}, A_{\infty}$ and a unique section, called $E$. Furthermore, $E \cap F_{X}$ being the only $B$-invariant section implies that $E \cap F_{X}$ is the unique curve of negative self-intersection in $F_{X}$ if $F_{X} \cong \Sigma_{m}$, $m>0$.

Application of the Algorithm: As a next step perform the sequence of elementary transformations given by the algorithm outlined in Figure 1. One must show that the algorithm stops. Since the center of the elementary transform intersects $F_{X}$ in a point not contained in $E$, i.e., not contained in the $\infty$-section of $F_{X}$, the self-intersection of $E \cap F_{X}$ in $F_{X}$ rises by one. Since it was negative when the algorithm started, it will eventually become zero, implying $F_{X} \cong \Sigma_{0}$, and the process terminates.

We claim that the point "Stop (B)" is never arrived at, i.e., $A_{0}$ and $A_{\infty}$ having only one $S$-invariant curve implies $F_{X} \cong \Sigma_{0}$. Note that $S$ has 
only one invariant curve in $A_{0}$ and $A_{\infty}$ if and only if $A_{0}, A_{\infty} \cong \Sigma_{0}$ and $S$ acts diagonally. This implies that $B_{U}$ has unique fixed points in $F_{X} \cap A_{0}$ and $F_{X} \cap A_{\infty}$, namely the intersection with $E$. Consequence: if $\sigma \subset F_{X}$ is a section not intersecting $E$ and $u \in B_{U} \backslash\{1\}$, then $\sigma, u . \sigma$ and $E \cap F_{X}$ are three mutually disjoint sections in $F_{X}$ over $F_{Y}$. Therefore $F_{X} \cong \Sigma_{0}$.

The Situation where the Algorithm stops: Let us now assume that the algorithm already stopped, i.e., $F_{X} \cong \Sigma_{0}$. Apply Lemma 3.7: as the algorithm terminates, the transformed variety is isomorphic to $Y \times_{Z} Y^{\prime}$. Now to say that there is a unique $B$-invariant section in $F_{X}$ over $F_{Y}$ which is not diagonal, it is equivalent to say that there exists a unique $S$-invariant curve in $Y^{\prime}$. Hence $Y^{\prime} \cong \Sigma_{0}$ and $S L_{2}$ acts diagonally. In particular, $X$ is the trivial bundle over $Y$ and $S L_{2}$ acts diagonally. Recall that this is the starting situation of Section 3.3.1.

End of the Proof: As a last step there is to prove that the inverses of the transformations we performed are the transformations used in Section 3.3.1, i.e., elementary transformations with center being $E_{0}$ or $E_{\infty}$. This, however, is clear if one takes into account that the algorithm transforms with centers being curves in $A_{0}$ or $A_{\infty}$ not intersecting $E$.

\subsubsection{Bundles over $\Sigma_{0}$}

The primary goal of this section is to characterize the diagonally twisted $\mathbb{P}_{1}$ bundles over $\Sigma_{0} \cong \mathbb{P}_{1} \times \mathbb{P}_{1}$. It is necessary to prove that sections which arise as closures of $S$-orbits are either disjoint or intersect transversally. The following lemma is a first step in this direction.

LEMMA 3.9. Let $B<S L_{2}$ be a Borel group and $\Sigma_{n}$ a Hirzebruchsurface with a surjection $\phi: \Sigma_{n} \rightarrow \mathbb{P}_{1}$. Assume that $B$ acts almost transitively on $\Sigma_{n}$ and that $\Sigma_{n}$ contains two $B$-invariant sections $\sigma_{1}$ and $\sigma_{2}$ over $\mathbb{P}_{1}$. Then either $\sigma_{1}$ and $\sigma_{2}$ are disjoint or they intersect transversally.

Proof. As a first step, remark that there are at most $2 B$-invariant sections in $\Sigma_{n}$. The existence of a third would contradict the almost transitive action, because if $\eta \in \mathbb{P}_{1}$ is a point in the open orbit, then it's isotropy group must act almost transitively on the fiber $X_{\eta}$ and fix the intersection with of $X_{\eta}$ all invariant sections. But there are no non-trivial automorphisms of a generic fiber fixing three points. This means that we only need to find two disjoint or transversal sections in order to prove the claim.

The same line of argument shows that $B$ may not have two fixed points on the base $\mathbb{P}_{1}$, for otherwise the unipotent part $U$ of $B$ would act trivially 
on $\mathbb{P}_{1}$. Thus, if $X_{\eta}$ is a general fiber, $U$ would stabilize $X_{\eta}$. But $U$ acts nontrivially on $\Sigma_{n}$, because $\Sigma_{n}$ and $B$ both have dimension 2. Consequently, $U$ would act non-trivially on $X_{\eta}$, and $X_{\eta}$ contains only one point which is invariant under the isotropy group $B_{\eta}$. So there would only be one invariant section.

We prove the lemma by induction

Start of Induction: $n=0$. Assume without loss of generality that $\phi$ : $\Sigma_{0} \cong \mathbb{P}_{1} \times \mathbb{P}_{1} \rightarrow \mathbb{P}_{1}$ is the projection onto the first factor. If the $B$-action on the second factor has two fixed points, then there are 2 disjoint sections, and we are finished. Otherwise, note that there is only one $B$-action on $\mathbb{P}_{1}$ with exactly one fixed point - up to isomorphy. Thus, after appropriate choice of coordinates, we can assume that $B$ acts diagonally on $\Sigma_{0}$. In this situation $B$ stabilizes the diagonal in $\Sigma_{0}$ and a fiber of the projection to the second factor. These curves meet transversally.

Step of Induction: Assume that the lemma is true for all numbers smaller than a given $n>0$. We will assume that the lemma is false for $n$ and derive a contradiction. Thus, suppose that we are given two $B$ invariant divisors $\sigma_{1}$ and $\sigma_{2}$ which do not intersect transversally. Let $\sigma_{1}$ be the unique curve of negative self-intersection in $\Sigma_{n}$, this curve is a section which is invariant under the full automorphism group. Let $F$ be the unique $B$-invariant $\phi$-fiber, the preimage of the $B$-fixed point in $\mathbb{P}_{1}$.

Claim: the group $B$ has two fixed points in $F$.

If the claim holds, then we can perform a $B$-equivariant elementary transformation where we choose the center to be the $B$-fixed point which is not contained in $\sigma_{1}$. If $X^{\prime}$ is the transformed variety and $\sigma_{1}^{\prime}$ and $\sigma_{2}^{\prime}$ are the strict transforms of $\sigma_{1}$ and $\sigma_{2}$, then $\sigma_{1}^{\prime}$ and $\sigma_{2}^{\prime}$ still intersect nontransversally: the intersection number $\sigma_{1}^{\prime} \cdot \sigma_{2}^{\prime}$ is even bigger than $\sigma_{1} \cdot \sigma_{2}$. On the other hand, by choice of the center, $X^{\prime} \cong \Sigma_{n-1}$. We obtain a contradiction to the induction hypothesis and are finished.

It remains to show the claim. Again assume to the contrary, i.e., assume that there was only one $B$-fixed point in $F$. Let $T<B$ be a torus. Since all $B$-actions on $\mathbb{P}_{1}$ which have only one fixed point are isomorphic, we know that $T$ acts on $F \cong \mathbb{P}_{1}$ with weight 2 . Similarly, $T$ acts on $\sigma_{1}$ with weight 2 ; this is because $\sigma_{1}$ is a section and the restricted map $\left.\phi\right|_{\sigma_{1}}: \sigma_{1} \rightarrow \mathbb{P}_{1}$ is equivariant. Now linearize the $T$-action at the intersection point $\sigma_{1} \cap F$. Realize that $F$ and $\sigma_{1}$ intersect transversally. But the only 2-dimensional $T$-representation space containing two $T$-invariant curves of weight 2 which additionally intersect transversally has weights $(2,2)$. Thus, 
any two $T$-invariant curves passing through the intersection point must intersect transversally. But since the intersection $\sigma_{1} \cap \sigma_{2}$ is $B$-fixed, $\sigma_{1} \cap \sigma_{2} \subset F$ so that $\sigma_{1}$ and $\sigma_{2}$ are two $T$-invariant curves passing through $\sigma_{1} \cap F$. This is absurd.

Proposition 3.10. (Characterization of the $X_{\Sigma_{0}, n}$ ) Under the Assumption 3.2, if $Y \cong \Sigma_{0}$ and $S$ acts almost transitively on $X$, then $X$ is a splitting bundle or one of the diagonally twisted bundles $X_{\Sigma_{0}, n}$ from Example 3.3.2.

Proof. To start with, choose a morphism $\pi: Y \rightarrow Z \times \mathbb{P}_{1}$ and define $F, F_{X}$ and $F_{Y}$ as in Notation 3.6. If $F_{X} \cong \Sigma_{0}$, we are finished by using Lemma 3.7. Thus, assume that $F_{X} \cong \Sigma_{n}, n>0$. No simple factor of $S$ acts trivially on $Y$. Thus either $S \cong S L_{2}$, acting diagonally on $Y$ or $S \cong S L_{2} \times S L_{2}$.

If $S \cong S L_{2} \times S L_{2}$, let $S^{\prime}<S$ be the factor of $S$ acting trivially on $Z$ and note that there are always two disjoint $S^{\prime}$-invariant sections $\sigma_{1}$ and $\sigma_{2}$ in $F_{X}$ over $F_{Y}$. If $S^{\prime \prime}$ is the other factor of $S$, then $S^{\prime \prime}$ must stabilize the locus where $S^{\prime}$ has 1-dimensional orbits; this is because $S^{\prime}$ and $S^{\prime \prime}$ commute. In particular, $S^{\prime \prime} . \sigma_{1}$ and $S^{\prime \prime} . \sigma_{2} \subset F_{X}$ are two disjoint sections over $Z$, displaying $X$ as a splitting Zariski bundle.

For the remainder of the proof consider the situation where $S \cong S L_{2}$. The isotropy $S_{\eta}$ at a generic point $\eta \in Y$ is a torus. This torus fixes two points in the associated $S_{\eta}$-invariant $\phi$-fiber $X_{\eta}$, and a standard argument shows that the closures of their $S$-orbits are rational sections. If these are disjoint, we can stop here. Thus, assume that they have non-trivial intersection. We claim that $X \cong X_{\Sigma_{0}, n}$.

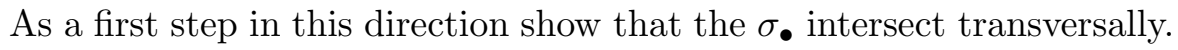
In order to see this, consider the stabilizer $B$ of $F_{X}$, which is a Borelsubgroup of $S$. The curves $\sigma_{1} \cap F_{X}$ and $\sigma_{2} \cap F_{X}$ are two $B$-invariant sections in $F_{X}$ over $F_{Y}$ which intersect in a single point. Lemma 3.9 claims that the intersection of these curves must be transversal. This transversality implies that the sections become disjoint if one performs an elementary transformation with center $\sigma_{1} \cap \sigma_{2}$. In other words, if $X^{\prime}$ is the transformed variety, then the strict transforms of $\sigma_{1}$ and $\sigma_{2}$ are disjoint. This already shows that $X^{\prime}$ is a splitting Zariski bundle.

The Triviality of the Bundle over the Diagonal: If $\Delta_{X}$ denotes the preimage of the $S$-invariant diagonal $\Delta \subset Y$, then $\Delta_{X}$ contains the center 
of the transformation and is transversal to both $\sigma_{1}$ and $\sigma_{2}$. Thus, after blowing up $\sigma_{1} \cap \sigma_{2}$, the strict transform of $\Delta_{X}$ becomes disjoint from the strict transforms of the $\sigma_{\bullet}$. This in turn implies that the exceptional divisor of the blow-up is isomorphic to $\Sigma_{0}$, and $S$ acts with one-dimensional orbits there. By construction, the same holds for the preimage of $\Delta$ in $X^{\prime}$. So $X^{\prime}$ is of the form $\mathbb{P}(\mathcal{O}(n,-n) \oplus \mathcal{O})$ since these are the only split $\mathbb{P}_{1}$-bundles which are trivial over the diagonal.

We have seen that the center of the back-transformation $X^{\prime} \rightarrow-\rightarrow$ is not contained in one of the $S$-invariant sections. So that back-transformation is exactly the construction performed in Example 3.3.2.

This establishes the isomorphy $X \cong X_{\Sigma_{0}, n}$ once we know that $n \neq 0$. Recall that $S L_{2}$ acts almost transitively on $X^{\prime}$. But if $n$ was 0 , then $X^{\prime} \cong$ $\Sigma_{0} \times \mathbb{P}_{1}$ was the trivial bundle and $S L_{2}$ having one-dimensional orbits over the diagonal would imply that $S L_{2}$ acts trivially on the second factor, a contradiction.

This proves $X \cong X_{\Sigma_{0}, n}$, and the claim is shown.

\subsubsection{Bundles over $\mathbb{P}_{2}$}

The classification of bundles over $\mathbb{P}_{2}$ is mainly a corollary of the classifications we have carried out already.

Proposition 3.11. Under the Assumption 3.2, if $Y \cong \mathbb{P}_{2}$ and $S$ acts almost transitively on $X$, then $X$ is isomorphic to a splitting $\mathbb{P}_{1}$-bundle, to the flag manifold $F_{(1,2)}(3)$, a blow-down of $X_{\Sigma_{1}, k_{0}, 0}$, or to a quotient of one of the relatively minimal varieties over $\Sigma_{0}$.

Proof. We tell between the possible $S$-actions on $Y$ :

$\boldsymbol{S} \cong \boldsymbol{S} \boldsymbol{L}_{\mathbf{3}}:$ If $S L_{3}$ acts transitively on $X$, then $X \cong F_{(1,2)}(3)$; this follows from the classification of the homogeneous manifolds. See [Win95]. Otherwise, the exceptional set $E$ is an unbranched cover of $\mathbb{P}_{2}$. A connected component of $E$ is a section, realizing $Y$ as a splitting Zariski bundle.

$\boldsymbol{S} \cong \boldsymbol{S} \boldsymbol{L}_{\mathbf{2}}$, and $\boldsymbol{S}$ has a fixed point $\mu \in \boldsymbol{Y}$ : Blow up the $\phi$-fiber $X_{\mu}$ an obtain a $\mathbb{P}_{1}$-bundle $X^{\prime} \rightarrow Y^{\prime} \cong \Sigma_{1}$. Let $E \subset X^{\prime}$ be the exceptional divisor of the blow-up. By Proposition 3.8, there are only two possibilities: 
If $X^{\prime}$ is a splitting Zariski bundle, then let $\sigma_{1}$ and $\sigma_{2}$ be two disjoint sections over $Y$. They intersect $E$ in two different fibers of the fibration $E \rightarrow X_{\mu}$. Consequence: the images of $\sigma_{1}$ and $\sigma_{2}$ in $X$ are disjoint sections, too. Thus $X$ is split.

If $X^{\prime} \cong X_{\Sigma_{1}, k_{0}, k_{\infty}}$, claim that $k_{\infty}=0$. As a first step, realize that $S$ acts non-trivially on $X_{\mu}$, or else a linearization argument would reveal that $S$ has only 2-dimensional orbits. Thus, $S$ acts diagonally on $E$, and there is exactly one $S$-invariant curve in $E$. This already shows that $k_{\infty}=0$, for otherwise $E$ had to contain two distinct $S$-invariant curves: the intersection with the unique $S$-invariant section over $Y^{\prime}$ and the center of the back-transformation $X_{\Sigma_{1}, k_{0}, k_{\infty}-1} \rightarrow X_{\Sigma_{1}, k_{0}, k_{\infty}}$.

$S \cong S L_{2}$, and $S$ does not have a fixed point in $Y$ : Recall that there exists an $S$-equivariant cover $\gamma: \Sigma_{0} \rightarrow \mathbb{P}_{2}$. The pull-back $X^{\prime}:=X \times_{Y}$ $\Sigma_{0}$ is $S$-quasihomogeneous. It was shown in Proposition 3.10 that $X^{\prime}$ is a splitting bundle or $X^{\prime} \cong X_{\Sigma_{0}, n}$, and $X$ is a quotient of $X^{\prime}$ by $\mathbb{Z}_{2}$.

\subsection{The Remaining Cases}

It remains to consider the cases where $S$ does not act almost transitively. We start with a classification of the varieties over $\Sigma_{n}$.

Proposition 3.12. Under the Assumption 3.2, if $Y \cong \Sigma_{n}$ and $S$ has generic orbits of dimension $\leq 2$, then $X$ is a splitting Zariski bundle.

Proof. If $S$ acts almost transitively on $Y$, then there is a subgroup $S^{\prime}<S$ acting almost transitively on $Y$ with $S^{\prime} \cong S L_{2}$. Hence, assume without loss of generality that $S \cong S L_{2}$. Choose $\pi: Y \rightarrow Z \cong \mathbb{P}_{1}$. If $F_{X} \cong \Sigma_{0}$, apply Lemma 3.7 and stop. Otherwise, let $B<S$ be the Borelgroup stabilizing $F_{X}$ and note that the generic $B$-orbit in $F_{X}$ has dimension at most 1 .

Claim: there are two disjoint $B$-invariant sections $\sigma_{0}, \sigma_{\infty}$ in $F_{X}$ over $F_{Y}$.

In order to prove the claim, let $T<B$ be a maximal torus. Recall that $T$ is not normal in $B$. Thus $T$ acts non-trivially on $F_{X}$, and a curve on $F_{X}$ is $B$-invariant if and only if it is $T$-invariant. Now the claim follows from the following fact: a maximal torus in $\operatorname{Aut}\left(\Sigma_{m}\right)$ always contains a subtorus whose fixed point set are two sections. 
Now we apply the claim: $D_{\bullet}:=S . \sigma_{\bullet}$ are disjoint sections in $X$ over $Y$, and we are finished.

If $S$ acts on $Y$ with 1-dimensional orbits, then choose $\pi: Y \rightarrow Z$ so that $S$ acts trivially on $Y$. Now there are two possibilities: the first is that $F_{X} \cong \Sigma_{m}$, where $m>0$. But then there are necessarily two disjoint sections in $F_{X}$ over $F_{Y}$. Recall that the set $D:=\{x \in X \mid \operatorname{dim} S . x \leq 1\}$ is closed. By what we saw above, $D$ is a $2: 1$ unbranched cover over $Y$. But $Y$ is simply connected. Thus, $D$ consists of 2 disjoint sections, and $X$ is a split Zariski bundle.

It remains to consider relatively minimal varieties over $\mathbb{P}_{2}$.

Proposition 3.13. In the setting of 3.2 , if $Y \cong \mathbb{P}_{2}$, and $S$ has generic orbits of dimension $\leq 2$, then $X$ is a splitting bundle.

Proof. Consider the different possibilities for the $S$-action on $Y$.

$\boldsymbol{S} \cong \boldsymbol{S L}_{\mathbf{3}}:$ All $S L_{3}$-orbits are isomorphic to $\mathbb{P}_{2}$ and, by $S$ acting transitively, are unbranched covers of $Y$. Three of them yield the identification with the trivial bundle.

$\boldsymbol{S} \cong \boldsymbol{S} \boldsymbol{L}_{2}$, and $\boldsymbol{S}$ has a fixed point $\mu \in \boldsymbol{Y}$ : We blow up the $\phi$-fiber $X_{\mu}$, obtain a $\mathbb{P}_{1}$-bundle over $\Sigma_{1}$ and apply Proposition 3.12. Argue as in the proof of Proposition 3.11 to see that $X$ is split as well.

$\boldsymbol{S} \cong \boldsymbol{S} \boldsymbol{L}_{2}$, and $\boldsymbol{S}$ does not have a fixed point in $\boldsymbol{Y}$ : Take a Borel group $B<S$. The isotropy $B_{\eta}$ of a generic point in $\eta \in Y$ is finite and cyclic. Hence there exists unique $B_{\eta}$-invariant point $f \in X_{\eta}$ and $D:=\overline{B . f}$ is a unique $S$-invariant section. The vanishing of all Ext-groups yields the claim.

\section{$\S 4$. Relatively Minimal Varieties over a Point}

Now we prove the classification Theorem 1.2 under the additional assumption that $Y$ is a point. The next lemma shows that nontrivial varieties occur only if the semi-simple part $S$ of $G$ is isomorphic to $S L_{2}$.

LEMMA 4.1. Under the assumption of Theorem 1.2, assume that $Y$ is a point. If $G$ contains a connected semi-simple group $S$ other than $S L_{2}$, then $X \cong \mathbb{P}_{3}, \mathbb{Q}_{3}$ or the weighted projective space $\mathbb{P}_{(1,1,1,2)}$. 
Proof. First assume that $X$ is singular and let $\tilde{X} \rightarrow X$ be an equivariant resolution of the singularities. By [Mor82, Cor. 3.6], there exists a relative contraction $\psi: \tilde{X} \rightarrow X^{\prime}$ over $X$. Note that $\psi$ must be divisorial. If $E$ is the exceptional divisor, use the classification of [Mor82, Thm. 3.3] to see that $E \cong \mathbb{P}_{2}, \mathbb{P}_{1} \times \mathbb{P}_{1}$ or a singular quadric. As the map $S \rightarrow \operatorname{Aut}(E)$ may not have a positive dimensional kernel, $S$ acts transitively on $E$. This already rules out the singular quadric. No $G$-invariant curve or divisor may intersect $E$. Consequently, the $G$-exceptional set in $X^{\prime}$ contains an isolated fixed point. By [HO80, Thm. 1 on p. 113], $X^{\prime}$ is a cone over a rational homogeneous surface. Again using the [Mor82, Thm. 3.3], only $\mathbb{P}_{3}$ and the blow down of the $\infty$-section of $\mathbb{P}\left(\mathcal{O}_{\mathbb{P}_{2}}(2) \oplus \mathcal{O}_{\mathbb{P}_{2}}\right)$ are possible. This variety is isomorphic to $\mathbb{P}_{(1,1,1,2)}$. By equality of the Picard-numbers, $X^{\prime}=X$.

If $X$ is smooth and homogeneous, then claim that $X \cong \mathbb{P}_{3}$ or $\mathbb{Q}_{3}$. If the complement of the open orbit has dimension $<2$, then use [HO80, Thm. 1 on p. 113 and Thm. 1 on p. 121] to yield the claim (the other varieties occurring in the classification are either not rational or have higher Picard-numbers). If the $G$-exceptional set contains a divisor $E$, then $S$ acts non-trivially on $E$, and $E \cong \mathbb{P}_{2}$ or $\mathbb{P}_{1} \times \mathbb{P}_{1}$. Now [Băd82, Thms. 1 and 5] apply, showing the claim.

As a next step we rule the possibility out that the generic $S$-orbit is a curve.

LEMMA 4.2. Under the assumption of Theorem 1.2, let $Y$ be a point. If $G$ contains a subgroup $S \cong S L_{2}$, then the generic $S$-orbit is of dimension 2 or 3 .

Proof. Assume to the contrary and let $C \subset X$ be any curve which is not $S L_{2}$-invariant. Then $D:=\overline{S L_{2} \cdot C}$ is an $S$-invariant divisor and the generic $S$-invariant curve does not intersect $D$. A contradiction to $X$ being minimal over a point.

The case that the generic $S L_{2}$-orbit is 2-dimensional has been classified in [Keb99]. The main result of that paper is that $X \cong \mathbb{Q}_{3}, \mathbb{P}_{3}, \mathbb{P}_{(1,1,1,2)}$ or $\mathbb{P}_{(1,1,2,3)}$.

The case that $S L_{2}$ acts almost transitively will be considered now. As a first step we recall that the assumption on $\mathbb{Q}$-factorial singularities already implies that $X$ is smooth. 
LEMMA 4.3. Let $X$ be a projective 3-dimensional variety with at most terminal singularities, quasihomogeneous with respect to an algebraic action of $S L_{2}$. Then either $X$ is smooth or not $\mathbb{Q}$-factorial.

Proof. If $X$ is not smooth, the singularities are isolated, hence fixed. Let $p \in X$ be a singular point. Recall that $X$ can equivariantly embedded into a projective space. Together with the complete reducibility of $S L_{2}$ representations this yields an embedding of a neighborhood $A$ of $p$ into a representation space $V$ such that $A$ is realized as the closure of an $S L_{2}$ orbit. A linearization argument using the assumption that $S L_{2}$ has a threedimensional orbit yields that $p$ is necessarily the unique fixed point in $A$; consequently, it's image is 0 .

Follow the proof [Kra85, Lemma 5 on p. 210] in order to construct two divisors $D_{1}, D_{2} \subset X$ with $D_{1} \cap D_{2}=\{p\}$.

Since $X$ is smooth, it must be contained in Iskovskih's list. It remains to identify those varieties which occur in our context.

Proposition 4.4. In the setting of Theorem 1.2, let $Y$ be a point. If $S=S L_{2}$ acts almost transitively on $X$, then $X$ is one of the following Fano-varieties: $\mathbb{P}_{3}, \mathbb{Q}_{3}, V_{22}^{S}$ or $V_{5}$.

Proof. We have already seen that $X$ is necessarily smooth. Let $T<S$ be a maximal torus, and let $F \subset X$ be the set of $T$-fixed points.

If $F$ is discrete, use a linearization argument to see that the Lefschetzindex of every fixed point is positive. The Borel fixed point theorem asserts that $F$ is not empty. Thus, $\chi(X)>0$ by the Hopf index theorem. We know already that $b_{0}=b_{6}=1, b_{1}=b_{5}=0$ as $X$ is rational and $b_{2}=b_{4}=1$ by the assumption that $\rho(X)=1$. Accordingly, $\chi(X)>0$ is possible iff $b_{3}<4$. The classification of Iskovskih implies already that only $\mathbb{P}_{3}, \mathbb{Q}_{3}, V_{5}$ and $V_{22}$ are possible. Recall that the only quasihomogeneous variety of type $V_{22}$ is the special $V_{22}^{S}$.

If $F$ is not discrete, then let $H$ be a component of $E$, the complement of the open $S$-orbit in $X$, such that $\operatorname{dim}(F \cap H)=1$. Since $S$ is 3-dimensional, $E$ is of pure dimension 2 and is the support of an effective divisor generating the anticanonical bundle $-K_{X}$. The $S$-action on $H$ cannot be almost transitive; instead, the generic $S$-orbit must be 1-dimensional. Furthermore, $X$ does not contain an $S$-fixed point, or else a linearization at this point would reveal a contradiction to the quasihomogeneous action of $S$, there 
being no 3-dimensional representation of $S L_{2}$ with 3-dimensional orbits. This implies already that the normalization $\tilde{H}$ of $H$ must be smooth. The closed and disjoint $S$-orbits realize $\tilde{H}$ as a product $\tilde{H} \cong C \times \mathbb{P}_{1}$, where $C$ is a smooth curve and $S$ acts on the second factor only. In particular, there is no isolated $T$-fixed point in $\tilde{H}$, and also none in $H$. As a next step, show that $H$ is smooth. In order verify this claim, note that $F \cap H$ is smooth and that every $S$-orbit in $H$ intersects $F \cap H$ transversally. If $H$ was singular, let $x \in H_{\text {sing }}$ be a $T$-fixed point. If $U<S L_{2}$ is a one-parameter group not fixing $x$, then by what we said above, the map

$$
\begin{aligned}
F \times U & \longrightarrow E \\
(f, u) & \longmapsto u . f
\end{aligned}
$$

has maximal rank at $(x, 1)$, a contradiction. The adjunction formula and the non-triviality of $K_{H}$ show that $H$ is Fano. So $H \cong \mathbb{P}_{1} \times \mathbb{P}_{1}$ and [Băd82, Thm. 5] yields the claim.

\section{REFERENCES}

[Băd82] L. Bădescu, On ample divisors, Nagoya Math. J., 86 (1982), 155-171.

[Fuj85] T. Fujita, On polarized manifolds whose adjoint bundles are not semipositive, In T. Oda, editor, Advanced Studies in Pure Mathematics, 10 (1985), North-Holland publishing company, 167-178.

[HO80] A. Huckleberry and E. Oeljeklaus, Classification Theorems for Almost Homogeneous Spaces, Institut Elie Cartan, 1980.

[Huc90] A. Huckleberry, Actions of groups of holomorphic transformations, In Barth, Narasimhan, and Gamkrelidze, editors, Several complex variables VI. Complex manifolds, 69 (1990), Encycl. Math. Sci., Springer-Verlag, 143-196.

[Isk83] V. Iskovskih, Algebraic threefolds with special regard to the problem of rationality, Proc. Int. Cong. Math. at Warszawa, 1 (1984), 733-746.

[KMM87] Y. Kawamata, K. Matsuda and K. Matsuki, Introduction to the minimal model problem, In T. Oda, editor, Advanced Studies in Pure Mathematics, 10 (1985), North-Holland publishing company, 283-360.

[Keb98] S. Kebekus, Simple models of quasihomogeneous projective 3-folds, available on the Internet at http://www.mathematik.uni-bielefeld.de/documenta, Doc. Math. J. DMV., 3 (1998), 15-26.

[Keb99] $\longrightarrow$ On the classification of 3-dimensional $S L_{2}(\mathbb{C})$-varieties, Nagoya Math. J., 157 (2000), ??-??.

[Kra85] H. Kraft, Geometrische Methoden in der Invariantentheorie, Aspekte der Mathematik, D1, Vieweg, 2, durchges. aufl. edition, 1985.

[LV83] D. Luna and T. Vust, Plongements d'espaces homogènes, Comment Math. Helv., 58 (1983), 186-245. 
[Mar73] M. Maruyama, On a family of algebraic vector bundles, In Number Theory, Algebraic Geometry and Commutative Algebra, in honor of Y. Akizuki (1973), Tokyo, Kinokuniya, 95-146.

[MJ90] L. Moser-Jauslin, Smooth embeddings of $S L(2)$ and $P G L(2)$, J. Algebra, 2(132) (1990), 384-405.

[Mor82] S. Mori, Threefolds whose canonical bundles are not numerically effective, Ann. of Math., 116 (1982).

[MU83] S. Mukai and H. Umemura, Minimal rational threefolds, In M. Raynaud and T. Shioda, editors, Algebraic geometry, Proc. Jap.-Fr. Conf., Tokyo and Kyoto 1982, Lect. Notes Math., 1016 (1983), Springer, 490-518.

[Mum66] D. Mumford, Lectures on curves on an algebraic surface, Princeton University Press, 1966.

[Rei83] M. Reid, Minimal models of canonical 3-folds, In Advanced studies in pure math., 1 (1983), Kinokuniya and North Holland, 131-180.

[Rei87] — Young person's guide to canonical singularities, Proceedings of Symposia in Pure Mathematics, 46, 1987.

[Win95] J. Winkelmann, The Classification of three-dimensional homogeneous complex manifolds, Lecture Notes in Mathematics, 1602, Springer, 1995.

Mathematisches Institut der Universität Bayreuth

95440 Bayreuth

Germany

FAX: +49(0)921/55-2785

stefan.kebekus@uni-bayreuth.de 